\title{
The genetics of interstitial lung diseases
}

\author{
Raphael Borie (10 ${ }^{1,2}$, Pierre Le Guen ${ }^{1,2}$, Mada Ghanem ${ }^{1,2}$, Camille Taillé ${ }^{1,2}$, \\ Clairelyne Dupin (10) ${ }^{1,2}$, Philippe Dieudé (i) ${ }^{2,3}$, Caroline Kannengiesser (10) ${ }^{2,4}$ and \\ Bruno Crestani ${ }^{1,2}$
}

Affiliations: 'Service de Pneumologie A, Hôpital Bichat, AP-HP, Paris, France. ${ }^{2}$ INSERM U1152, Paris, France. ${ }^{3}$ Département de Génétique, Hôpital Bichat, AP-HP, Paris, France. ${ }^{4}$ Service de Rhumatologie, Hôpital Bichat, AP-HP, Paris, France.

Correspondence: Bruno Crestani, Service de Pneumologie A, Hôpital Bichat, 46 rue Henri Huchard, 75877 Paris Cedex 18, France. E-mail: bruno.crestanidaphp.fr

@ERSpublications

Common and rare genetic variants contribute to the development and clinical manifestation of many interstitial lung diseases http://bit.ly/31loHLh

Cite this article as: Borie R, Le Guen P, Ghanem M, et al. The genetics of interstitial lung diseases. Eur Respir Rev 2019; 28: 190053 [https://doi.org/10.1183/16000617.0053-2019].

ABSTRACT Interstitial lung diseases (ILDs) are a set of heterogeneous lung diseases characterised by inflammation and, in some cases, fibrosis. These lung conditions lead to dyspnoea, cough, abnormalities in gas exchange, restrictive physiology (characterised by decreased lung volumes), hypoxaemia and, if progressive, respiratory failure. In some cases, ILDs can be caused by systemic diseases or environmental exposures. The ability to treat or cure these ILDs varies based on the subtype and in many cases lung transplantation remains the only curative therapy. There is a growing body of evidence that both common and rare genetic variants contribute to the development and clinical manifestation of many of the ILDs. Here, we review the current understanding of genetic risk and ILD.

\section{Introduction}

There is growing evidence that genetic factors contribute to the development of interstitial lung disease (ILD), notably in a context of familial aggregation (i.e. familial interstitial pneumonia (FIP)). Idiopathic pulmonary fibrosis (IPF) is the most common and most severe form of ILD, and has thus received the most attention in pulmonary research. High-resolution computed tomography (HRCT) of the chest in IPF patients shows interstitial fibrosis, described as the usual interstitial pneumonia (UIP) pattern (figure 1). Patients typically progress to hypoxaemia and respiratory failure, with most patients dying from the disease within 5 years of diagnosis $[1,2]$. There are no curative therapies, but two drugs (nintedanib and pirfenidone) have been shown to slow disease progression $[3,4]$. Lung transplantation is feasible for only a small percentage of IPF patients.

The incidence of IPF is approximately 20 per 100000 males and 13 per 100000 females [5], but rising. Most individuals present aged 50-75 years. Many individuals can be diagnosed with UIP findings on HRCT of the chest, but when expert HRCT review is not definitive, patients are referred to lung biopsy for diagnosis. IPF patients have lung pathology consistent with a UIP pattern, characterised by interstitial fibrosis, honeycomb changes, fibroblastic foci and a paucity of inflammation [6,7]. While the aetiology of IPF remains unknown, genetic discoveries in monogenic familial forms of the disease over the past three decades have led to significant insights into the role of inherited risk mutations in disease pathogenesis and in the understanding of the intimate mechanisms of lung fibrosis.

Provenance: Publication of this peer-reviewed article was sponsored by Boehringer Ingelheim, Germany (principal sponsor European Respiratory Review issue 153).

Received: 24 May 2019 | Accepted after revision: 01 Aug 2019

Copyright $\odot$ ERS 2019. This article is open access and distributed under the terms of the Creative Commons Attribution Non-Commercial Licence 4.0. 

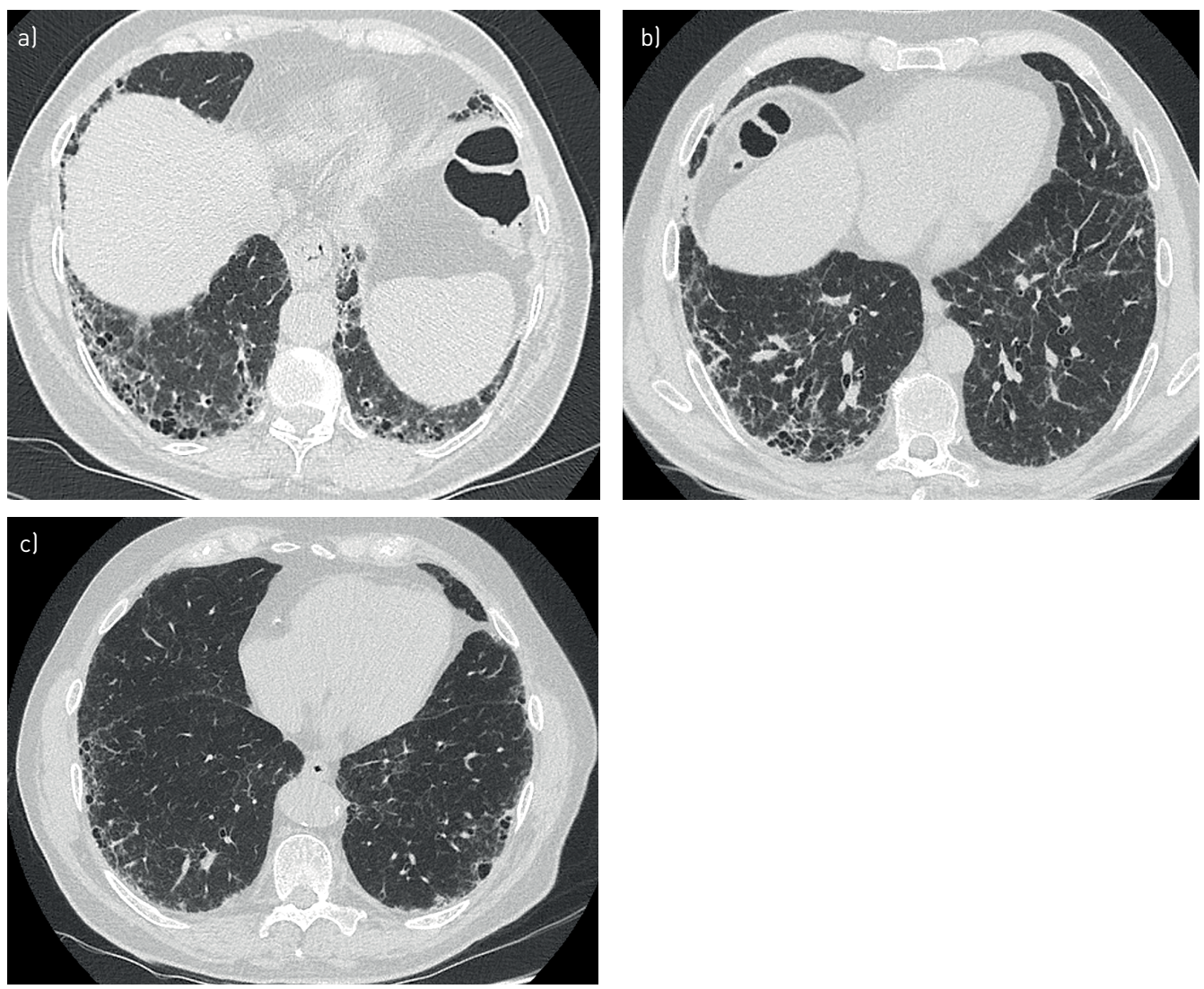

FIGURE 1 High-resolution computed tomography imaging of usual interstitial pneumonia: a) a patient with idiopathic pulmonary fibrosis (IPF) and carrier of rs35705950 within MUC5B, b) a patient with IPF and carrier of a TERC mutation and c) a patient with rheumatoid arthritis-interstitial lung disease and carrier of rs35705950.

Methods for genetic testing are rapidly evolving and allow for several genes to be analysed together. From targeted next-generation sequencing (NGS) panels to whole-genome sequencing (WES), each approach has its own advantages and disadvantages that should be specifically considered. Briefly, for patients with suspected inherited pulmonary fibrosis, most genetic laboratories are using targeted NGS panels analysing three to 300 selected genes altogether, although a few laboratories perform WES. Sanger sequencing is still used for familial study or confirmation of the presence of a genetic variation.

\section{Familial interstitial pneumonia}

Although there is no consensus definition, in the research setting FIP is usually defined as a case of ILD in which the patient also has a family history of two or more relatives with ILD $[8,9]$. Early studies suggested that familial forms of the disease accounted for $2-4 \%$ of IPF $[10,11]$, although later evidence suggests that this percentage may be higher [12,13]. Adults with FIP are essentially indistinguishable from sporadic IPF patients in terms of clinical presentation, radiographic findings and histopathology, except that those with FIP tend to present at earlier age [14].

A study of 111 families with FIP, including 309 individuals with ILD and 360 unaffected relatives, revealed that male sex $(55.7 \%$ versus $37.2 \% ; \mathrm{p}<0.0001)$, age $(68.3$ versus 53.1 years; $\mathrm{p}<0.0001)$ and having ever smoked cigarettes $(67.3 \%$ versus $34.1 \%$; $\mathrm{p}<0.0001)$ were risk factors for developing ILD. A UIP pattern was identified in $85 \%$ of patients; however, pathological heterogeneity was observed within individual families: $45 \%$ of these families having two or more pathological patterns identified within the affected individuals, with numerous families having evidence of UIP and non-specific interstitial pneumonia (NSIP) histopathology [14], an observation consistent with previous findings [15, 16], suggesting that distinct ILD categorisations may share similar pathogenesis pathways. The identification of cigarette smoking as a FIP risk factor also suggested that the interplay between genetic predisposition and environmental exposures is central to familial disease [14]. Many analyses of FIP families have suggested an autosomal dominant mode of inheritance with incomplete penetrance $[11,14,17]$. 
Common variants associated with ILD

Common variants associated with ILD are listed in table 1.

\section{MUC5B}

In 2011, SeiBold et al. [9] identified that a single nucleotide polymorphism rs35705950 is located in the promoter region of $M U C 5 B$, which encodes mucin 5B, a glycosylated macromolecular component of mucus, and is expressed in the normal bronchiolar epithelium. Using case-control analysis of non-Hispanic Whites, SEIBold et al. [9] observed that subjects heterozygous and homozygous for the $\mathrm{T}$ risk allele had increased ORs for disease of 6.8 (95\% CI 3.9-12.0) and 20.8 (95\% CI 3.8-113.7) for FIP and 9.0 (95\% CI 6.2-13.1) and 21.8 (95\% CI 5.1-93.5) for IPF, respectively. This initial study found a similar rs35705950 minor allele frequency between FIP and sporadic IPF individuals (0.338 and 0.375 , respectively), suggesting a similar genetic contribution of the $M U C 5 B$ risk variant in sporadic and familial IPF [9]. Microscopy of diseased lung also reveals that MUC5B protein is found in the honeycomb cyst, a characteristic pathological finding of UIP, the pattern consistent with IPF [18]

Numerous groups have replicated the association between MUC5B rs35705950 and IPF, identifying this variant as the strongest and most well-replicated single genetic risk factor for IPF [9, 19-25]. The rs35705950 T risk allele is common and was detected in 10\% of a non-Hispanic White control population [26]. The presence of the variant alone is insufficient to cause disease and approximately half of subjects with IPF do not carry this variant. The rs35705950 variant is neither necessary nor sufficient to cause disease, suggesting the involvement of other genetic or environmental factors to cause disease development; this remains an area of active research [27].

The MUC5B promoter polymorphism also appears to be specific to the risk of UIP and then most frequently associated with IPF, but eventually also associated with a UIP pattern in the context of hypersensitivity pneumonitis or rheumatoid arthritis (RA)-ILD [28, 29]. Indeed, in a study including 620 RA-ILD patients, 614 RA without ILD patients and 5448 unaffected controls, the MUC5B risk polymorphism was associated with the risk of ILD in RA patients when compared with unaffected controls or with RA without ILD patients. Interestingly, the increased risk of ILD was restricted to patients with a UIP pattern (41\% of the whole RA-ILD group): UIP RA-ILD versus RA-no ILD (OR 6.1, 95\% CI 2.9-13.1; $\mathrm{p}=2.5 \times 10^{-6}$ ) and non-UIP RA-ILD versus RA-no ILD (OR 1.3, 95\% CI 0.6-2.8; $\mathrm{p}=0.46$ ) [29].

Moreover, in two cohorts of 145 and 72 Caucasian chronic hypersensitivity pneumonitis patients, the MUC5B risk polymorphism prevalence was $24.4 \%$ and $32.3 \%$, respectively, versus $10.7 \%$ in the general population [28]. Among the 189 patients with chronic hypersensitivity pneumonitis and CT scan

TABLE 1 Common variants associated with idiopathic pulmonary fibrosis

\begin{tabular}{|c|c|c|}
\hline Gene & Single nucleotide polymorphism(s) & Key reference(s) \\
\hline AKAP13 & rs62025270 & [39] \\
\hline ATP11A & rs1278769 & [31] \\
\hline CDKN1A & rs2395655, rs733590 & {$[105]$} \\
\hline DPP9 & rs12610495 & [31] \\
\hline$D S P$ & rs2076295 & [31] \\
\hline ELMOD2 & Unknown & [106] \\
\hline FAM13A & rs2609255 & [31] \\
\hline HLA-DRB1 & rs2395655 & {$[31]$} \\
\hline ILIRN & rs408392, rs419598, rs2637988 & {$[49,105]$} \\
\hline IL8 & rs4073, rs2227307 & {$[107,108]$} \\
\hline MAPT & rs1981997 & [31] \\
\hline MDGA2 & rs7144383 & [21] \\
\hline MUC2 & rs7934606 & {$[31]$} \\
\hline MUC5B & rs35705950 & {$[9,20,21,31,40,49]$} \\
\hline OBFC1 & rs11191865 & [31] \\
\hline SPPL2C & rs17690703 & {$[21]$} \\
\hline TERC & rs6793295 & [31] \\
\hline TERT & rs2736100 & {$[31,49,109]$} \\
\hline TGFB1 & rs1800470 & [21] \\
\hline TLR3 & rs3775291 & [110] \\
\hline TOLLIP & rs111521887, rs5743894, rs5743890 & {$[21]$} \\
\hline TP53 & rs12951053, rs12602273 & [105] \\
\hline
\end{tabular}


available, the MUC5B risk polymorphism was associated with the presence of traction bronchiectasis, suggestive of probable UIP $(\mathrm{p}<0.001)$, but not with a pattern consistent with definite or possible UIP or presence of radiographic honeycombing [28]. rs35705950 has not been associated with ILD in systemic sclerosis [20,22], sarcoidosis [20] or inflammatory myositis, or with chronic obstructive pulmonary disease or asthma [30]. A subsequent genome-wide association study (GWAS) that examined numerous genetic loci as well as rs35705950 in a fibrotic idiopathic interstitial pneumonia (IIP) cohort that largely contained IPF subjects, but also contained other forms of fibrotic IIP, confirmed the association between the MUC5B genotype and the fibrotic IIP phenotype [31].

rs35705950 has also been associated with a risk of IPF in Hispanic White and Asian populations, although the overall rs35705950 frequency is low in Asian populations [24, 25, 32]. IPF is thought to be rare in African populations [33].

\section{Other common genetic variants and IPF}

Although the common $M U C 5 B$ promoter polymorphism is the most widely and well-studied common genetic variant associated with IPF and FIP, other common variants have been discovered through GWAS as high-throughput variant screening methods have developed.

In 2008, researchers from Japan identified an association of a common TERT (telomerase reverse transcriptase) variant with susceptibility to IPF [34]. In 2013, a large GWAS confirmed several known disease-associated loci (chromosome 5p15 which contains TERT; $11 \mathrm{p} 15$ which contains MUC5B; 3q25 near TERC (telomerase RNA component)) and identified seven new loci, including FAM13A (family with sequence similarity 13 member A; 4q22), DSP (desmoplakin; 6p24), OBFC1 (oligonucleotide-binding fold containing 1; 10q24), ATP11A (ATPase phospholipid transporting 11A; 13q4), DPP9 (dipeptidyl peptidase 9; 19p13), and risk loci on chromosomes 7q22 and 15q14-15 [31]. The implicated genes span a wide variety of biological functions, but could be categorised into the following: host defence (MUC5B and ATP11A), cell-cell adhesion (DSP and DPP9) and DNA repair (TERT, TERC and OBFC1) [31, 35-37]. It has been estimated that these loci, excluding the MUC5B variant, may account for up to a third of disease risk, emphasising the importance of genetic predisposition in fibrotic ILD [31, 37].

Another IPF GWAS performed by an independent group replicated the MUC5B rs35705950 association, but also suggested the contribution of additional risk alleles located in TOLLIP (Toll-interacting protein) and SPPL2C (signal peptidase-like 2C). Importantly, this study not only identified risk variants, but also drew connections between specific variants (rs5743890) in TOLLIP and differential mortality from disease [21]. However, a recent study suggested that rs5743890 in TOLLIP was not associated with increased risk of IPF when adjusted for the presence of other genetic risk factors such as MUC5B [38].

Another GWAS identified a new locus associated with IPF near AKAP13 (A-kinase anchoring protein 13; rs62025270, OR 1.27, 95\% CI 1.18-1.37; $\mathrm{p}=1.32 \times 10^{-9}$ ). The allele associated with increased susceptibility to IPF was also associated with increased expression of AKAP13 mRNA in control lung tissue [39]. Interestingly, as was observed in the initial MUC5B promoter polymorphism study [9], the odds ratios for loci identified by the 2013 GWAS by FingerLIN et al. [31] did not differ between FIP and sporadic IPF cases, reinforcing that both diseases share a common genetic background.

\section{Disease severity}

Retrospective analyses of large clinical trials data reveals that IPF patients with the minor allele $(\mathrm{T})$ at rs35705950 in MUC5B had improved survival when compared with wild-type (GG) subjects of the same cohort [40], suggesting that the MUC5B promoter variant identifies a subset of patients with IPF who have a distinct phenotype/prognosis. Similarly, genotype at the variant in TOLLIP first associated with IPF by Noтн et al. [21] (rs5743890) is also associated with differential survival and may be associated with a differential response to $\mathrm{N}$-acetylcysteine [41].

A post hoc analysis of the CAPACITY and ASCEND trials showed that patients with the MUC5B risk allele were older (68.1 versus 65.5 years) and had a slower disease progression than patients without the risk allele. Pirfenidone was, however, still associated with a decreased decline of forced vital capacity (FVC) [42]. Nintedanib has not been examined in terms of efficacy by genotype.

\section{Rare variants associated with ILD}

Numerous Mendelian disorders can be associated with ILD. Rare variants associated with ILD are listed in table 2. Here, we will focus on the most frequent causes: surfactant-associated protein gene mutations and telomere-related genes. 


\section{Surfactant proteins}

\section{SFTPC}

Surfactant protein C (SPC) is one of four surfactant proteins expressed in the alveoli and functions to alter surface tension to prevent alveolar collapse. This protein is expressed throughout the lung epithelium during lung development, but in the mature lung it is localised to type II alveolar epithelial cells [43]. Early studies of genetic risk in the development of IPF used FIP subjects. The first disease-associated genetic variants were identified in surfactant protein genes among FIP patients [15, 44-46]. These studies identified heterozygous mutations in SFTPC coding for SPC [15, 44], which segregated with diseased subjects and was not found to be present in controls.

Both paediatric and adult ILD have been linked to SFTPC mutations [15]. Although SFTPC mutations were first linked to paediatric cases of ILD, the contribution of SFTPC mutations in adult FIP has also been established. In 2002, Thomas et al. [16] described a family in which 11 adults had ILD, six with biopsy-confirmed UIP/IPF and five with clinical diagnoses of IPF, as well as three paediatric cases of NSIP. In vitro studies also revealed that the L188Q SFTPC mutation results in a pro-SPC molecule that cannot be folded properly, prompting endoplasmic reticulum stress and caspase pathway activation [47, 48]. Subsequently, additional mutations in SFTPC have been found in other FIP cohorts, up to 25\% of FIP cases in a Dutch cohort, although this is a lot higher than what has been observed elsewhere $[49,50]$.

SFTPC mutations are rarely found in sporadic IPF cases. Interestingly, de novo mutations are frequent in children and may explain up to $50 \%$ of cases [51].

Other surfactant-related genes

Heterozygous mutations in SFTPA2 (surfactant protein A2) or SFTPA1 (surfactant protein A1) have been identified in subjects with FIP and/or lung adenocarcinoma [46, 52].

ATP-binding cassette transporter A3 (ABCA3) is expressed in type II alveolar epithelial cell lamellar bodies and is important in surfactant processing. Although homozygous $A B C A 3$ mutations are usually associated with respiratory failure in newborns [53], one teenage ILD patient with a UIP pattern and one 41-year-old patient with combined pulmonary fibrosis and emphysema (CPFE) carrying mutations of $A B C A 3$ have been reported $[54,55]$. Other studies have suggested that in infant ILD, those with heterozygous SFTPC mutations and concomitant heterozygous mutations in $A B C A 3$ may be more likely to develop clinical ILD [56]. Therefore, ABCA3 recessive mutations may modify the effects of SFTPC dominant mutations [57].

NKX2-1 (NK2 homeobox 1) encodes a transcription factor closely related to surfactant protein transcription [58]. Heterozygous mutations are classically associated with the triad of ILD, hypothyroidism and neurological anomalies (hypotonia, delayed development and chorea) [59]. These mutations may be

\begin{tabular}{|c|c|c|}
\hline Phenotype & Genels) & Key reference(s) \\
\hline IPF, DKC & $\begin{array}{c}\text { TERT, TERC, TINF2, PARN, NAF1, } \\
\text { RTEL1, DKC1 }\end{array}$ & [111] \\
\hline IPF, lung cancer, CPFE, alveolar proteinosis & SFTPA1, SFTPA2, SFTPC, ABCA3 & {$[49,50,52,56]$} \\
\hline Lung brain thyroid syndrome & $N K X 2.1($ TITF1) & {$[1,59]$} \\
\hline Hermansky-Pudlak syndrome & HPS1-HSP8, AP3B1 & [112] \\
\hline Alveolar proteinosis & CSF2RA, CSF2RB, GATA2, MARS & [113-115] \\
\hline Lysinuric protein intolerance & SLC7A7 & [116] \\
\hline Pulmonary alveolar microlithiasis & SLC34A2 & [117] \\
\hline Gaucher's disease & GBA & [118] \\
\hline Niemann-Pick disease & SMPD1 & [119] \\
\hline Fabry disease & GLA & {$[120]$} \\
\hline $\begin{array}{l}\text { STING-associated vasculopathy with onset } \\
\text { in infancy }\end{array}$ & TMEM173, COPA & [121-123] \\
\hline Poikilodermia lung fibrosis & FAM111B & [124] \\
\hline Acadian variant of Fanconi syndrome & NDUFAF6 & {$[125]$} \\
\hline Prolidase deficiency & PEPD & [126] \\
\hline
\end{tabular}

IPF: idiopathic pulmonary fibrosis; DKC: dyskeratosis congenita; CPFE: combined pulmonary fibrosis and emphysema; STING: stimulator of interferon genes. 
associated with ILD without hypothyroidism and neurological anomalies in up to a third of cases, including adult cases in which the most common HRCT pattern is atypical for UIP [59].

Biallelic ABCA3 mutations and heterozygous NKX2-1, SFTPA1 SFTPA2 and SFTPC mutations in adults may share similar clinical and radiological presentation. The most frequent radiological pattern associates predominant diffuse ground-glass opacities, septal thickening and cysts of variable size with a preferential distribution in the upper lobes and in subpleural areas (figure 2). Differentiating emphysema from cysts is sometimes difficult and SFTPC mutation must be evoked in a young patient presenting CPFE [60]. However, at a later stage of disease, honeycombing can predominate.

Histologically, the most frequently related pattern in adults is UIP, although NSIP, organising pneumonia or desquamative interstitial pneumonia have also been reported. Moderate inflammation and centrolobular fibrosis can be observed [50].

In children, successful treatments reported in case reports or short series include methylprednisolone, hydroxychloroquine or azithromycin [61-63]. No treatment appears to reduce disease in a patient with predominant honeycombing lesions. The effect of antifibrotic drugs, such as pirfenidone or nintedanib, is to date unknown. The disease does not appear to recur after pulmonary transplantation [61].

\section{Telomere-related genes}

Telomeres are regions of noncoding repetitive nucleotide repeats (TTAGGG) at the ends of chromosomes that protect them from deterioration during mitosis or fusion with neighbouring chromosomes. The telomerase complex is the group of proteins and RNA that catalyses the addition of these nucleotide repeats to the ends of chromosomes. There are numerous components to the telomerase complex, including TERT and TERC, which are essential for normal operation and telomere integrity. Shortening of telomeres has been associated with numerous disease manifestations, as have mutations in telomere-related genes [64], including ILD. Indeed, numerous studies of FIP cases and their kindred have identified
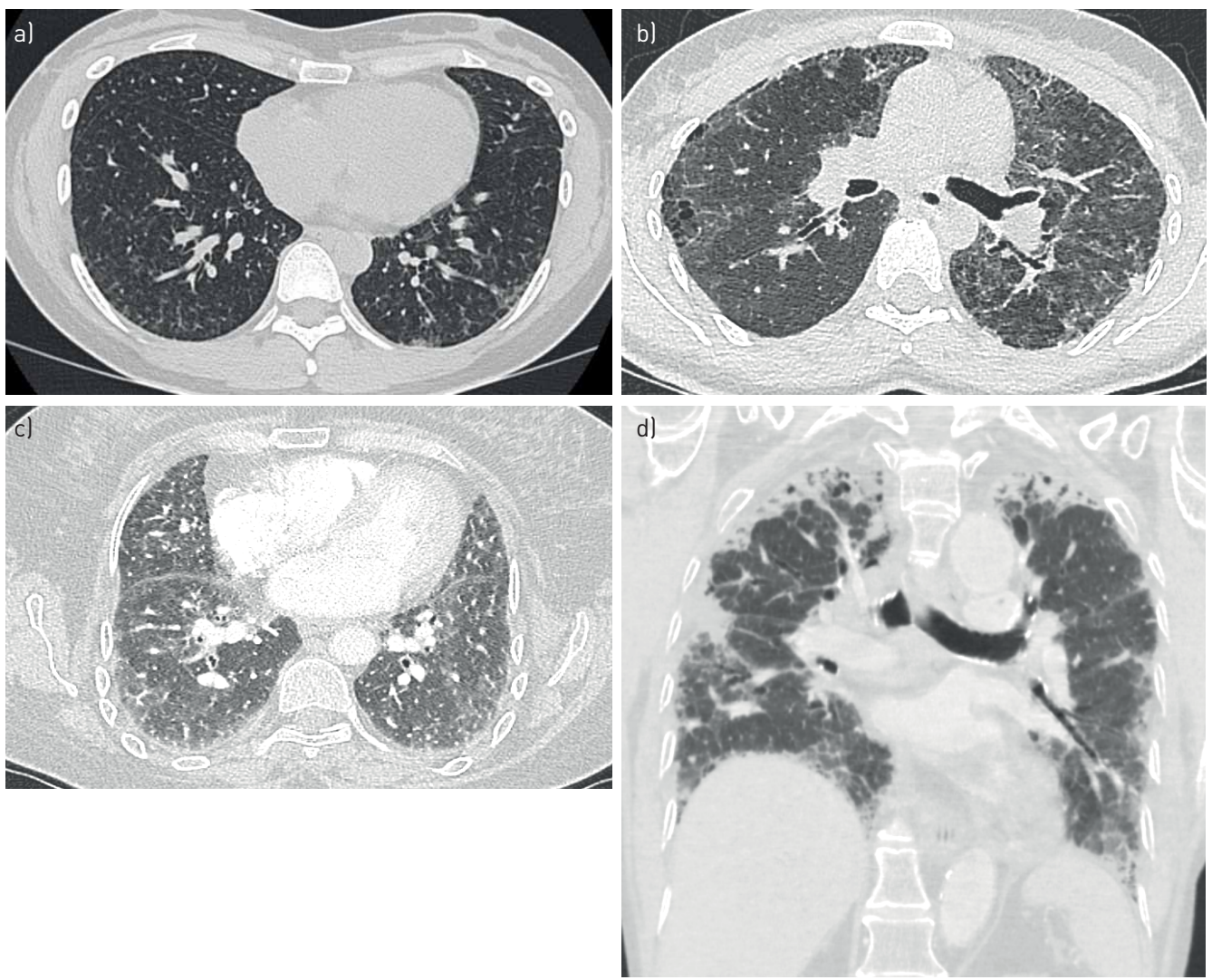

FIGURE 2 High-resolution computed tomography of a) non-usual interstitial pneumonia (UIP) pattern (indeterminate) with ground-glass opacities and reticulation associated with a SFTPA1 mutation, and b, c) non-UIP pattern (indeterminate) with ground-glass opacities and cysts from two patients, both carriers of a compound heterozygous $A B C A 3$ mutation. d) Pattern suggestive of pleuroparenchymal fibroelastosis associated with a TERT mutation. 
mutations in various telomere-related genes (TERT, TERC, RTEL1 (regulator of telomere elongation helicase 1), PARN (poly(A)-specific RNase), NAF1 (nuclear assembly factor 1 ribonucleoprotein), DKC1 (dyskerin pseudouridine synthase 1) and TINF2 (TERF1 interacting nuclear factor 2)). For instance, TERT and TERC mutations have been identified in up to a sixth of pulmonary fibrosis families $[65,66]$.

Dyskeratosis congenita (DKC) is a diagnosis made based on a triad of abnormal skin pigmentation, nail dystrophy and oral leukoplakia, but can affect numerous organ systems [67], including the bone marrow. Pulmonary fibrosis is found in $20 \%$ of cases and respiratory failure is the most common proximal cause of death in these patients. In X-linked DKC, mutations in DKC1 are causative [68, 69], but some autosomal dominant forms of DKC are linked to mutations in TERT and TERC [70-72]. In 2005, ARMANIOs et al. [72] reported a TERT mutation in a family affected by DKC in which pulmonary fibrosis was the dominant clinical finding.

Subsequently, Armanios et al. [72] and TSAKIRI et al. [66] identified heterozygous TERT and TERC mutations. In vitro examination of the mutations demonstrated decreased telomerase activity and that peripheral blood leukocyte telomere lengths were shorter in mutation carriers when compared with age-matched non-carriers. These studies suggested that telomere-related gene mutations cause disease in $\sim 15 \%$ of FIP.

ARMAnios et al. [72], TSAKIRI et al. [66] and others also examined telomere length itself and its relationship to pulmonary fibrosis, independent of mutations in TERT and TERC [73, 74]. CRONKHITE et al. [73] analysed a cohort of pulmonary fibrosis patients without TERT and TERC, including probands from 59 families with FIP and 73 subjects with sporadic IPF. They found that $24 \%$ of FIP subjects and $23 \%$ of sporadic IPF subjects had evidence of telomere shortening, with peripheral blood leukocyte telomere lengths below the 10th percentile compared with age-matched controls. ALDER et al. [74] analysed 100 cases of sporadic IPF, and found one subject with a TERC mutation and no mutations in TERT. 62 of these subjects had their telomere lengths measured in peripheral blood lymphocytes and $97 \%$ showed telomere lengths shorter than the median in healthy controls; furthermore, $10 \%$ had telomere lengths shorter than the first percentile of healthy controls.

AlDER et al. [74] found cryptogenic cirrhosis in a few of the IPF subjects, which prior to their publication had only been described in the setting of DKC. These additional findings suggested that, at least in a small subset of patients, "telomeropathy", or a syndrome in which multiple organs are affected by telomere shortening, may be present. A subsequent study that examined this link further sequenced numerous subjects with both aplastic anaemia and pulmonary fibrosis, and found that the concurrence of these two disorders (both separately associated with telomere dysfunction) was highly predictive for the presence of germline telomerase mutation [75, 76], a finding that could affect the clinical evaluation and decision making for those contemplating bone marrow or lung transplantation.

More recent studies have utilised WES techniques to discover rare variants in other telomerase pathway genes. Specifically, this technique has been utilised to pinpoint rare variants in the RTEL1 and PARN genes found to be associated with FIP [77-79]. As in the case of other telomerase pathway genes, affected subjects with the identified genetic variants in these genes had evidence of shortened peripheral blood leukocyte telomeres [77, 78], although the mechanism through which PARN mutations affect telomere length remains poorly understood [80]. Exome sequencing has also identified rare TINF2 and NAF1 mutations in FIP $[81,82]$. Additionally, a novel $D K C 1$ mutation was also recently described in association with FIP [83].

Mechanistically, although the specific link between telomere-related gene mutations and pulmonary fibrosis remains an area of active research, in vivo studies utilising mouse models for loss of function of telomere-related genes suggest that when these genes dysfunction, the lung epithelia's response to injury is impaired [84].

Heterozygous mutations have been detected in familial forms of pulmonary fibrosis involving TERT ( 15\%), RTEL1 (5-10\%), PARN ( 5\%) and TERC ( 3\%). Mutations in DKC1, NAF1 and TINF2 are much rarer $[76-79,81,83,85,86]$. Telomere-related gene mutations may be found in 1-9\% of sporadic IPF cases $[42,87]$. None of these genes is the site of a frequent mutation and new genetic variants are continually being identified. The penetrance (risk of pulmonary fibrosis developing in a telomere-related gene mutation carrier) depends on several factors, including environmental exposure [76].

Telomeres shorten from generation to generation in patients with TERT, TERC or RTEL1 mutations because of transmission of the short telomeres independent of transmission of the mutation [86]. As telomere shortening varies depending on the involved gene and its impact on telomere length, genetic anticipation may be more pronounced for carriers of TERC than PARN mutations [86]. 
The disease phenotype of patients carrying telomerase pathway mutations is varied. In individuals with known telomerase pathway mutations, the prevalence of ILD increases with age, as illustrated by a study of TERT mutation carriers in which none of the subjects $<40$ years of age had evidence of ILD, yet its prevalence in those $>60$ years of age was $60 \%$ [88]. An observational study of 115 pulmonary fibrosis patients with telomerase pathway mutations (TERC, TERT, RTEL1 and PARN) was conducted and found that TERC mutation carriers were diagnosed at an earlier age (mean 51 years) relative to the other study subjects (58 years for TERT, 60 years for RTEL1 and 65 years for PARN) [86].

Pulmonary fibrosis and extrapulmonary manifestations associated with telomerase complex mutations are variably called "telomere disease", "telomeropathy" or "short telomere syndrome", with lack of a consensual definition. In patients with TERT mutation and pulmonary fibrosis, the classical triad of DKC is usually absent. However, $15-40 \%$ of mutation carriers present premature hair greying (before 30 years of age) $[76,89]$. In patients with telomere-related gene mutations and pulmonary fibrosis, anaemia is present in $17-27 \%$, macrocytosis in $24-41 \%$ and thrombocytopenia in $8-54 \%[76,86,88]$. DKC1, TINF2 and TERC mutation carriers seem more prone to the development of haematological involvement than TERT or RTEL1 mutation carriers (our own observation and [86]). Patients can also present liver cirrhosis: cryptogenic, viral or alcoholic. Elevated liver enzyme levels or liver involvement was reported in 5-27\% of patients with ILD and telomere-related gene mutations [76, 86]. GoRGY et al. [90] highlighted the high frequency of hepatopulmonary syndrome associated with telomere-related gene mutations in a retrospective series of nine patients without ILD. Among the six patients with available liver biopsies, the most common abnormality was nodular regenerative hyperplasia (in four patients) [90].

A typical UIP pattern on chest CT was initially reported in up to $74 \%$ of cases, but was recently found in only $46-55 \%$ of cases (figure 1) $[76,86,88]$. Unusual features found in $13-20 \%$ of cases included upper lung predominance of fibrosis, centrolobular fibrosis or a pleuro-parenchymal fibroelastosis pattern (figure 2) [76, 86, 88, 91]. Taken together, $14-40 \%$ of cases show a CPFE pattern [92].

The decline of pulmonary function (FVC) of patients with ILD associated with telomerase complex mutations seems unexpectedly high when compared with placebo arms of IPF clinical trials (130-210 mL per year) [93]. NEWTON et al. [86] reported a $300 \mathrm{~mL}$ per year decline of FVC whatever the gene involved (TERC, TERT, RTEL1 or PARN) and the ILD entity (IPF or not).

The safety and effectiveness of pirfenidone has been reported in patients with telomere-related gene variants. One European retrospective study was not able to show an effect of pirfenidone on lung function decline, with a decline of FVC of $161.8 \pm 31.2 \mathrm{~mL}$ per year before and $235.0 \pm 49.7 \mathrm{~mL}$ per year after pirfenidone initiation [94]. However, a post hoc analysis of two phase 3 clinical trials (CAPACITY and ASCEND) identified 102 patients with IPF as carriers of rare telomere-related gene variants. Although carriers of a rare variant within TERT, PARN, TERC or RTEL1 had a more rapid decline in predicted FVC than patients without a rare variant ( $1.66 \%$ versus $0.83 \%$ per month), pirfenidone still reduced the decline of FVC in this subgroup of severe patients [42]. No data are available for nintedanib.

Danazol, a synthetic sex hormone with androgenic properties, showed promise for pulmonary fibrosis associated with telomere disease, with stabilisation of diffusing capacity of the lung for carbon monoxide, FVC and CT scan findings during a 2-year treatment period [95].

Given the young age of most patients, lung transplantation is often discussed. At least five retrospective series reported the outcome of lung transplantation in 61 telomere-related gene mutation carriers [96-100]. Most patients required adjustment of immunosuppression because of haematological toxicity. Thrombocytopenia and a need for platelet transfusion were frequent, and myelodysplastic syndrome and/ or bone marrow failure occurred in some patients. Acute kidney failure requiring dialysis support seemed unexpectedly frequent (up to 50\%) [96, 97]. Interestingly, short telomeres and mutations of telomere-related genes have been associated with increased prevalence of cytomegalovirus (CMV) infection after lung transplantation [100]. Very recently, in a cohort of 262 patients who received lung transplantation, patients with TERT, RTEL1 or PARN mutations $(\mathrm{n}=31(11.8 \%))$ were reported to have a reduced post-transplantation survival (hazard ratio 1.82, 95\% CI 1.07-3.08; $\mathrm{p}=0.03$ ) and higher risk of chronic lung allograft dysfunction (hazard ratio 2.88, 95\% CI 1.42-5.87; p=0.004) [99]. However, this retrospective study did not report higher risk of haematological complication or renal insufficiency in telomere-related gene mutation carriers [99].

In an independent cohort, patients with telomere length below the 10th percentile before transplant were reported to have a worse survival and also a shorter time to onset of chronic lung allograft dysfunction [101]. Comparison of the less than 10th percentile telomere length group with the greater than 10th percentile telomere length group showed a higher rate of primary graft dysfunction, but there were no differences in the incidence of acute rejection, cytopenias, infection or renal dysfunction [101]. 
However, in some patients, telomere-related gene mutations have also been associated with a risk of immunodeficiency with a spontaneous risk of opportunistic infection such as Pneumocystis jirovecii or after lung transplantation as assessed by an increased risk of CMV infection [100, 102].

\section{Combined rare and common variants}

The studies described in detail in the previous sections have generally taken the approach of examining either common variants or rare variants and their relationship to IIP or IPF risk [76, 103]. Very recently, a combined analysis of rare and common variants of 1510 patients with IPF showed 1046 patients (69.2\%) were carriers of the rs35705950 (MUC5B) risk allele, but only $30(3 \%)$ of them were also carriers of a rare variant within TERT, whereas 34 (7\%) of the non-carriers of rs35705950 were also carriers of a rare variant within TERT [42]. Furthermore, in a recently reported study of 3624 IPF patients and 4442 controls, deep targeted resequencing of candidate genes showed that TERT and RTEL1 were independently associated with the risk of IPF [38].

\section{Conclusions}

Although these initial studies suggest that genetic variants could be useful in assisting with making a prognosis, the relationships between genotype at different variants and survival are still being investigated and need to be validated in prospective studies. Future therapeutic trials will need to take into account phenotypic and genotypic variation to allow for a deeper understanding of how these characteristics can and should be integrated into shared decision making. At present, given the limited data definitively linking genetic variants with concrete clinical outcomes or therapeutic responses, sequencing and genotyping patients are not part of routine IPF or fibrotic IIP care. Our point actually is to consider genetic analysis (including telomere-related gene and MUC5B sequencing) and telomere length for familial pulmonary fibrosis, short telomere syndrome, and sequencing surfactant genes for cryptogenic pulmonary fibrosis below the age of 50 years. Evidence of a pathogenic mutation should at least lead to genetic counselling while awaiting targeted therapy [104].

Conflict of interest: R. Borie reports personal fees and non-financial support from Roche, and Boehringer Ingelheim, outside the submitted work. P. Le Guen has nothing to disclose. M. Ghanem has nothing to disclose. C. Taillé has received personal fees and other funding from AstraZeneca and Roche, personal fees from Teva and Genzyme, grants, personal fees and other from GlaxoSmithKline, Novartis and Sanofi, and other funding from Boehringer Ingelheim. C. Dupin reports personal fees, non-financial support and other from AstraZeneca, Boehringer, GlaxoSmithKline and Novartis, personal fees and other from Chiesi, personal fees from Sanofi, and non-financial support and other from Roche, outside the submitted work. P. Dieudé has nothing to disclose. C. Kannengiesser has nothing to disclose. B. Crestani reports personal fees from AstraZeneca, grants, personal fees and non-financial support from Boehringer Ingelheim and Roche, personal fees and non-financial support from Sanofi, and grants from Novartis, outside the submitted work.

\section{References}

1 Bjoraker JA, Ryu JH, Edwin MK, et al. Prognostic significance of histopathologic subsets in idiopathic pulmonary fibrosis. Am J Respir Crit Care Med 1998; 157: 199-203.

2 Ley B, Collard HR, King TE. Clinical course and prediction of survival in idiopathic pulmonary fibrosis. Am J Respir Crit Care Med 2011; 183: 431-440.

3 King TE, Bradford WZ, Castro-Bernardini S, et al. A phase 3 trial of pirfenidone in patients with idiopathic pulmonary fibrosis. N Engl J Med 2014; 370: 2083-2092.

4 Richeldi L, du Bois RM, Raghu G, et al. Efficacy and safety of nintedanib in idiopathic pulmonary fibrosis. N Engl J Med 2014; 370: 2071-2082.

5 Hutchinson J, Fogarty A, Hubbard R, et al. Global incidence and mortality of idiopathic pulmonary fibrosis: a systematic review. Eur Respir J 2015; 46: 795-806.

6 Lynch DA, Sverzellati N, Travis WD, et al. Diagnostic criteria for idiopathic pulmonary fibrosis: a Fleischner Society White Paper. Lancet Respir Med 2018; 6: 138-153.

7 Raghu G, Collard HR, Egan JJ, et al. An Official ATS/ERS/JRS/ALAT Statement: idiopathic pulmonary fibrosis: evidence-based guidelines for diagnosis and management. Am J Respir Crit Care Med 2011; 183: 788-824.

8 Kropski JA, Pritchett JM, Zoz DF, et al. Extensive phenotyping of individuals at risk for familial interstitial pneumonia reveals clues to the pathogenesis of interstitial lung disease. Am J Respir Crit Care Med 2015; 191: 417-426.

9 Seibold MA, Wise A, Speer M, et al. A common MUC5B promoter polymorphism and pulmonary fibrosis. N Engl J Med 2011; 364: 1503-1512.

10 Hodgson U, Laitinen T, Tukiainen P. Nationwide prevalence of sporadic and familial idiopathic pulmonary fibrosis: evidence of founder effect among multiplex families in Finland. Thorax 2002; 57: 338-342.

11 Marshall RP, Puddicombe A, Cookson WO, et al. Adult familial cryptogenic fibrosing alveolitis in the United Kingdom. Thorax 2000; 55: 143-146.

12 Lawson WE, Loyd JE. The genetic approach in pulmonary fibrosis: can it provide clues to this complex disease? Proc Am Thorac Soc 2006; 3: 345-349.

13 Loyd JE. Pulmonary fibrosis in families. Am J Respir Cell Mol Biol 2003; 29: S47-S50. 
14 Steele MP, Speer MC, Loyd JE, et al. Clinical and pathologic features of familial interstitial pneumonia. Am J Respir Crit Care Med 2005; 172: 1146-1152.

15 Nogee LM, Dunbar AE, Wert SE, et al. A mutation in the surfactant protein C gene associated with familial interstitial lung disease. N Engl J Med 2001; 344: 573-579.

16 Thomas AQ, Lane K, Phillips J, et al. Heterozygosity for a surfactant protein C gene mutation associated with usual interstitial pneumonitis and cellular nonspecific interstitial pneumonitis in one kindred. Am J Respir Crit Care Med 2002; 165: 1322-1328.

17 Lee H-L, Ryu JH, Wittmer MH, et al. Familial idiopathic pulmonary fibrosis: clinical features and outcome. Chest 2005; 127: 2034-2041.

18 Seibold MA, Smith RW, Urbanek C, et al. The idiopathic pulmonary fibrosis honeycomb cyst contains a mucocilary pseudostratified epithelium. PLoS One 2013; 8: e58658.

19 Zhang Y, Noth I, Garcia JGN, et al. A variant in the promoter of MUC5B and idiopathic pulmonary fibrosis NT5E mutations and arterial calcifications. N Engl J Med 2011; 364: 1576-1577.

20 Stock CJ, Sato H, Fonseca C, et al. Mucin 5B promoter polymorphism is associated with idiopathic pulmonary fibrosis but not with development of lung fibrosis in systemic sclerosis or sarcoidosis. Thorax 2013; 68: 436-441.

21 Noth I, Zhang Y, Ma S-F, et al. Genetic variants associated with idiopathic pulmonary fibrosis susceptibility and mortality: a genome-wide association study. Lancet Respir Med 2013; 1: 309-317.

22 Borie R, Crestani B, Dieude P, et al. The MUC5B variant is associated with idiopathic pulmonary fibrosis but not with systemic sclerosis interstitial lung disease in the European Caucasian population. PLoS One 2013; 8: e70621.

23 Wei R, Li C, Zhang M, et al. Association between MUC5B and TERT polymorphisms and different interstitial lung disease phenotypes. Transl Res 2014; 163: 494-502.

24 Horimasu Y, Ohshimo S, Bonella F, et al. MUC5B promoter polymorphism in Japanese patients with idiopathic pulmonary fibrosis. Respirology 2015; 20: 439-444

25 Pelito AL, Selman M, Kim DS, et al. The MUC5B promoter polymorphism is associated with idiopathic pulmonary fibrosis in a Mexican cohort but is rare among Asian ancestries. Chest 2015; 147: 460-464.

26 Hunninghake GM, Hatabu H, Okajima Y, et al. MUC5B promoter polymorphism and interstitial lung abnormalities. N Engl J Med 2013; 368: 2192-2200.

27 Mathai SK, Newton CA, Schwartz DA, et al. Pulmonary fibrosis in the era of stratified medicine. Thorax 2016; 368: 2192-2200.

28 Ley B, Newton CA, Arnould I, et al. The MUC5B promoter polymorphism and telomere length in patients with chronic hypersensitivity pneumonitis: an observational cohort-control study. Lancet Respir Med 2017; 5: 639-647.

29 Juge P-A, Lee JS, Ebstein E, et al. MUC5B promoter variant and rheumatoid arthritis with interstitial lung disease. N Engl J Med 2018; 379: 2209-2219.

$30 \quad$ Yang IV, Schwartz DA. Epigenetics of idiopathic pulmonary fibrosis. Transl Res 2015; 165: 48-60.

31 Fingerlin TE, Murphy E, Zhang W, et al. Genome-wide association study identifies multiple susceptibility loci for pulmonary fibrosis. Nat Genet 2013; 45: 613-620.

32 Wang $\mathrm{C}$, Zhuang Y, Guo W, et al. Mucin 5B promoter polymorphism is associated with susceptibility to interstitial lung diseases in Chinese males. PLoS One 2014; 9: e104919.

33 Ley B, Collard HR. Epidemiology of idiopathic pulmonary fibrosis. Clin Epidemiol 2013; 5: 483-492.

34 Mushiroda T, Wattanapokayakit S, Takahashi A, et al. A genome-wide association study identifies an association of a common variant in TERT with susceptibility to idiopathic pulmonary fibrosis. J Med Genet 2008; 45: 654-656.

35 Yang IV, Fingerlin TE, Evans CM, et al. MUC5B and idiopathic pulmonary fibrosis. Ann Am Thorac Soc 2015; 12: Suppl. 2, S193-S199.

36 Mathai SK, Schwartz DA, Warg LA. Genetic susceptibility and pulmonary fibrosis. Curr Opin Pulm Med 2014; 20: 429-435.

37 Mathai SK, Yang IV, Schwarz MI, et al. Incorporating genetics into the identification and treatment of idiopathic pulmonary fibrosis. BMC Med 2015; 13: 191

38 Moore C, Blumhagen RZ, Yang IV, et al. Resequencing study confirms host defense and cell senescence gene variants contribute to the risk of idiopathic pulmonary fibrosis. Am J Respir Crit Care Med 2019; 200: 199-208.

39 Allen RJ, Porte J, Braybrooke R, et al. Genetic variants associated with susceptibility to idiopathic pulmonary fibrosis in people of European ancestry: a genome-wide association study. Lancet Respir Med 2017; 5: 869-880.

40 Peljto AL, Zhang Y, Fingerlin TE, et al. Association between the MUC5B promoter polymorphism and survival in patients with idiopathic pulmonary fibrosis. JAMA 2013; 309: 2232-2239.

41 Oldham JM, Ma SF, Martinez FJ, et al. TOLLIP, MUC5B, and the response to $N$-acetylcysteine among individuals with idiopathic pulmonary fibrosis. Am J Respir Crit Care Med 2015; 192: 1475-1482.

42 Dressen A, Abbas AR, Cabanski C, et al. Analysis of protein-altering variants in telomerase genes and their association with $M U C 5 B$ common variant status in patients with idiopathic pulmonary fibrosis: a candidate gene sequencing study. Lancet Respir Med 2018; 6: 603-614.

43 Cameron HS, Somaschini M, Carrera P, et al. A common mutation in the surfactant protein $\mathrm{C}$ gene associated with lung disease. J Pediatr 2005; 146: 370-375.

44 Lawson WE, Grant SW, Ambrosini V, et al. Genetic mutations in surfactant protein C are a rare cause of sporadic cases of IPF. Thorax 2004; 59: 977-980.

45 Fernandez BA, Fox G, Bhatia R, et al. A Newfoundland cohort of familial and sporadic idiopathic pulmonary fibrosis patients: clinical and genetic features. Respir Res 2012; 13: 64.

46 Wang Y, Kuan PJ, Xing C, et al. Genetic defects in surfactant protein A2 are associated with pulmonary fibrosis and lung cancer. Am J Hum Genet 2009; 84: 52-59.

47 Mulugeta S, Maguire JA, Newitt JL, et al. Misfolded BRICHOS SP-C mutant proteins induce apoptosis via caspase-4- and cytochrome c-related mechanisms. Am J Physiol Lung Cell Mol Physiol 2007; 293: L720-L729.

48 Lawson WE, Crossno PF, Polosukhin VV, et al. Endoplasmic reticulum stress in alveolar epithelial cells is prominent in IPF: association with altered surfactant protein processing and herpesvirus infection. Am J Physiol Lung Cell Mol Physiol 2008; 294: L1119-L1126. 
van Moorsel CHM, ten Klooster L, van Oosterhout MFM, et al. SFTPA2 mutations in familial and sporadic idiopathic interstitial pneumonia. Am J Respir Crit Care Med 2015; 192: 1249-1252.

van Moorsel CHM, van Oosterhout MFM, Barlo NP, et al. Surfactant protein C mutations are the basis of a significant portion of adult familial pulmonary fibrosis in a Dutch cohort. Am J Respir Crit Care Med 2010; 182: 1419-1425.

Kröner C, Reu S, Teusch V, et al. Genotype alone does not predict the clinical course of SFTPC deficiency in paediatric patients. Eur Respir J 2015; 46: 197-206.

Nathan N, Giraud V, Picard C, et al. Germline SFTPA1 mutation in familial idiopathic interstitial pneumonia and lung cancer. Hum Mol Genet 2016; 25: 1457-1467.

Wambach JA, Casey AM, Fishman MP, et al. Genotype-phenotype correlations for infants and children with ABCA3 deficiency. Am J Respir Crit Care Med 2014; 189: 1538-1543.

Young LR, Nogee LM, Barnett B, et al. Usual interstitial pneumonia in an adolescent with $A B C A 3$ mutations. Chest 2008; 134: 192-195.

Epaud R, Delestrain C, Louha M, et al. Combined pulmonary fibrosis and emphysema syndrome associated with ABCA3 mutations. Eur Respir J 2014; 43: 638-641.

Bullard JE, Nogee LM. Heterozygosity for $A B C A 3$ mutations modifies the severity of lung disease associated with a surfactant protein C gene (SFTPC) mutation. Pediatr Res 2007; 62: 176-179.

Crossno PF, Polosukhin VV, Blackwell TS, et al. Identification of early interstitial lung disease in an individual with genetic variations in ABCA3 and SFTPC. Chest 2010; 137: 969-973.

Hamdan H, Liu H, Li C, et al. Structure of the human Nkx2.1 gene. Biochim Biophys Acta 1998; 1396: 336-348.

Nattes E, Lejeune S, Carsin A, et al. Heterogeneity of lung disease associated with NK2 homeobox 1 mutations. Respir Med 2017; 129: 16-23.

Cottin V, Reix P, Khouatra C, et al. Combined pulmonary fibrosis and emphysema syndrome associated with familial SFTPC mutation. Thorax 2011; 66: 918-919.

Kroner C, Wittmann T, Reu S, et al. Lung disease caused by ABCA3 mutations. Thorax 2016; 72: 213-220.

Mechri M, Epaud R, Emond S, et al. Surfactant protein C gene (SFTPC) mutation-associated lung disease: high-resolution computed tomography (HRCT) findings and its relation to histological analysis. Pediatr Pulmonol 2010; 45: 1021-1029.

Thouvenin G, Abou Taam R, Flamein F, et al. Characteristics of disorders associated with genetic mutations of surfactant protein C. Arch Child 2010; 95: 449-454.

Armanios M, Blackburn EH. The telomere syndromes. Nat Rev Genet 2012; 13: 693-704.

Cogan JD, et al. Telomerase mutations in families with idiopathic pulmonary fibrosis. N Engl J Med 2007; 356: 1317-1326.

Tsakiri KD, Cronkhite JT, Kuan PJ, et al. Adult-onset pulmonary fibrosis caused by mutations in telomerase. Proc Natl Acad Sci USA 2007; 104: 7552-7557.

Vulliamy T, Dokal I. Dyskeratosis congenita. Semin Hematol 2006; 43: 157-166.

of DC. Br J Haematol 1998; 103: 990-996.

Heiss NS, Knight SW, Vulliamy TJ, et al. X-linked dyskeratosis congenita is caused by mutations in a highly conserved gene with putative nucleolar functions. Nat Genet 1998; 19: 32-38.

Vulliamy T, Marrone A, Szydlo R, et al. Disease anticipation is associated with progressive telomere shortening in families with dyskeratosis congenita due to mutations in TERC. Nat Genet 2004; 36: 447-449.

Vulliamy T, Marrone A, Goldman F, et al. The RNA component of telomerase is mutated in autosomal dominant dyskeratosis congenita. Nature 2001; 413: 432-435.

Armanios M, Chen J-L, Chang Y-PC, et al. Haploinsufficiency of telomerase reverse transcriptase leads to anticipation in autosomal dominant dyskeratosis congenita. Proc Natl Acad Sci USA 2005; 102: 15960-15964.

Cronkhite JT, Xing C, Raghu G, et al. Telomere shortening in familial and sporadic pulmonary fibrosis. Am J Respir Crit Care Med 2008; 178: 729-737.

Alder JK, Chen JJ-L, Lancaster L, et al. Short telomeres are a risk factor for idiopathic pulmonary fibrosis. Proc Natl Acad Sci USA 2008; 105: 13051-13056.

Parry EM, Alder JK, Qi X, et al. Syndrome complex of bone marrow failure and pulmonary fibrosis predicts germline defects in telomerase. Blood 2011; 117: 5607-5611.

Borie R, Tabèze L, Thabut G, et al. Prevalence and characteristics of TERT and TERC mutations in suspected genetic pulmonary fibrosis. Eur Respir J 2016; 48: 1721-1731.

Stuart BD, Choi J, Zaidi S, et al. Exome sequencing links mutations in PARN and RTEL1 with familial pulmonary fibrosis and telomere shortening. Nat Genet 2015; 47: 512-517.

Cogan JD, Kropski JA, Zhao M, et al. Rare variants in RTEL1 are associated with familial interstitial pneumonia. Am J Respir Crit Care Med 2015; 191: 646-655.

Kannengiesser C, Borie R, Menard C, et al. Heterozygous RTEL1 mutations are associated with familial pulmonary fibrosis. Eur Respir J 2015; 46: 474-485. telomerase RNA component. Nat Genet 2015; 47: 1482-1488.

Alder JK, Stanley SE, Wagner CL, et al. Exome sequencing identifies mutant TINF2 in a family with pulmonary fibrosis. Chest 2015; 147: 1361-1368.

Stanley SE, Gable DL, Wagner CL, et al. Loss-of-function mutations in the RNA biogenesis factor NAF1 predispose to pulmonary fibrosis-emphysema. Sci Transl Med 2016; 8: 351ra107.

Kropski JA, Mitchell DB, Markin C, et al. A novel dyskerin (DKC1) mutation is associated with familial interstitial pneumonia. Chest 2014; 146: e1-e7.

Alder JK, Barkauskas CE, Limjunyawong N, et al. Telomere dysfunction causes alveolar stem cell failure. Proc Natl Acad Sci USA 2015; 112: 5099-5104.

Fukuhara A, Tanino Y, Ishii T, et al. Pulmonary fibrosis in dyskeratosis congenita with TINF2 gene mutation. Eur Respir J 2013; 42: 1757-1759.

Newton CA, Batra K, Torrealba J, et al. Telomere-related lung fibrosis is diagnostically heterogeneous but uniformly progressive. Eur Respir J 2016; 48: 1710-1720. 
Petrovski S, Todd JL, Durheim MT, et al. An exome sequencing study to assess the role of rare genetic variation in pulmonary fibrosis. Am J Respir Crit Care Med 2017; 196: 82-93.

88 Diaz de Leon A, Cronkhite JT, Katzenstein AL, et al. Telomere lengths, pulmonary fibrosis and telomerase (TERT) mutations. PLoS One 2010; 5: e10680.

89 Diaz de Leon A, Cronkhite JT, Yilmaz C, et al. Subclinical lung disease, macrocytosis, and premature graying in kindreds with telomerase (TERT) mutations. Chest 2011; 140: 753-763.

90 Gorgy AI, Jonassaint NL, Stanley SE, et al. Hepatopulmonary syndrome is a frequent cause of dyspnea in the short telomere disorders. Chest 2015; 148: 1019-1026.

91 Nunes H, Jeny F, Bouvry D, et al. Pleuroparenchymal fibroelastosis associated with telomerase reverse transcriptase mutations. Eur Respir J 2017; 49: 1602022.

92 Nunes H, Monnet I, Kannengiesser C, et al. Is telomeropathy the explanation for combined pulmonary fibrosis and emphysema syndrome? Report of a family with TERT mutation. Am J Respir Crit Care Med 2014; 189: 753-754.

93 Karimi-Shah BA, Chowdhury BA. Forced vital capacity in idiopathic pulmonary fibrosis - FDA review of pirfenidone and nintedanib. N Engl J Med 2015; 372: 1189-1191.

94 Justet A, Thabut G, Manali E, et al. Safety and efficacy of pirfenidone in patients carrying telomerase complex mutation. Eur Respir J 2018; 51: 1701875.

95 Townsley DM, Dumitriu B, Young NS. Bone marrow failure and the telomeropathies. Blood 2014; 124: 2775-2783.

96 Borie R, Kannengiesser C, Hirschi S, et al. Severe hematologic complications after lung transplantation in patients with telomerase complex mutations. J Heart Lung Transplant 2015; 34: 538-546.

97 Silhan LL, Shah PD, Chambers DC, et al. Lung transplantation in telomerase mutation carriers with pulmonary fibrosis. Eur Respir J 2014; 44: 178-187.

98 Tokman S, Singer JP, Devine MS, et al. Clinical outcomes of lung transplant recipients with telomerase mutations. J Heart Lung Transplant 2015; 34: 1318-1324.

99 Swaminathan AC, Neely ML, Frankel CW, et al. Lung transplant outcomes in pulmonary fibrosis patients with telomere-related gene variants. Chest 2019; 156: 477-485.

100 Popescu I, Mannem H, Winters SA, et al. Impaired CMV immunity in idiopathic pulmonary fibrosis lung transplant recipients with short telomeres. Am J Respir Crit Care Med 2018; 199: 362-376.

101 Newton CA, Kozlitina J, Lines JR, et al. Telomere length in patients with pulmonary fibrosis associated with chronic lung allograft dysfunction and post-lung transplantation survival. J Heart Lung Transplant 2017; 36: 845-853.

102 Borie R, Kannengiesser C, Sicre de Fontbrune F, et al. Pneumocystosis revealing immunodeficiency secondary to TERC mutation. Eur Respir J 2017; 50: 1701443.

103 van der Vis JJ, Snetselaar R, Kazemier KM, et al. Effect of Muc5b promoter polymorphism on disease predisposition and survival in idiopathic interstitial pneumonias. Respirology 2016; 21: 712-717.

104 Borie R, Kannengiesser C, Sicre de Fontbrune F, et al. Management of suspected monogenic lung fibrosis in a specialised centre. Eur Respir Rev 2017; 26: 28446600.

105 Korthagen NM, van Moorsel CH, Barlo NP, et al. Association between variations in cell cycle genes and idiopathic pulmonary fibrosis. PLoS One 2012; 7: e30442.

106 Pulkkinen V, Bruce S, Rintahaka J, et al. ELMOD2, a candidate gene for idiopathic pulmonary fibrosis, regulates antiviral responses. FASEB J 2010; 24: 1167-1177.

107 Ahn M-H, Park B-L, Lee S-H, et al. A promoter SNP rs4073T >A in the common allele of the interleukin 8 gene is associated with the development of idiopathic pulmonary fibrosis via the IL- 8 protein enhancing mode. Respir Res 2011; 12: 73 .

108 Richards TJ, Park C, Chen Y, et al. Allele-specific transactivation of matrix metalloproteinase 7 by FOXA2 and correlation with plasma levels in idiopathic pulmonary fibrosis. Am J Physiol Lung Cell Mol Physiol 2012; 302: L746-L754.

109 Stuart BD, Lee JS, Kozlitina J, et al. Effect of telomere length on survival in patients with idiopathic pulmonary fibrosis: an observational cohort study with independent validation. Lancet Respir Med 2014; 2: 557-565.

110 O'Dwyer DN, Armstrong ME, Trujillo G, et al. The Toll-like receptor 3 L412F polymorphism and disease progression in idiopathic pulmonary fibrosis. Am J Respir Crit Care Med 2013; 188: 1442-1450.

111 Borie R, Bouvry D, Cottin V, et al. Regulator of telomere length 1 (RTEL1) mutations are associated with heterogeneous pulmonary and extra-pulmonary phenotypes. Eur Respir J 2018; 53: 1800508.

112 El-Chemaly S, Young LR. Hermansky-Pudlak syndrome. Clin Chest Med 2016; 37: 505-511.

113 Suzuki T, Sakagami T, Rubin BK, et al. Familial pulmonary alveolar proteinosis caused by mutations in CSF2RA. J Exp Med 2008; 205: 2703-2710.

114 Griese M, Zarbock R, Costabel U, et al. GATA2 deficiency in children and adults with severe pulmonary alveolar proteinosis and hematologic disorders. BMC Pulm Med 2015; 15: 87.

115 Hadchouel A, Wieland T, Griese M, et al. Biallelic mutations of methionyl-tRNA synthetase cause a specific type of pulmonary alveolar proteinosis prevalent on Reunion Island. Am J Hum Genet 2015; 96: 826-831.

116 Mauhin W, Habarou F, Gobin S, et al. Update on lysinuric protein intolerance, a multi-faceted disease retrospective cohort analysis from birth to adulthood. Orphanet J Rare Dis 2017; 12: 3.

117 Corut A, Senyigit A, Ugur SA, et al. Mutations in SLC34A2 cause pulmonary alveolar microlithiasis and are possibly associated with testicular microlithiasis. Am J Hum Genet 2006; 79: 650-656.

118 Mistry PK, Lopez G, Schiffmann R, et al. Gaucher disease: progress and ongoing challenges. Mol Genet Metab 2017; 120: 8-21.

119 Schuchman EH. The pathogenesis and treatment of acid sphingomyelinase-deficient Niemann-Pick disease. J Inherit Metab Dis 2007; 30: 654-663.

120 Svensson CK, Feldt-Rasmussen U, Backer V. Fabry disease, respiratory symptoms, and airway limitation - a systematic review. Eur Clin Respir J 2015; 2: 26721.

121 Liu Y, Jesus AA, Marrero B, et al. Activated STING in a vascular and pulmonary syndrome. $N$ Engl J Med 2014; 371: 507-518 
122 Picard C, Thouvenin G, Kannengiesser C, et al. Severe pulmonary fibrosis as the first manifestation of interferonopathy (TMEM173 mutation). Chest 2016; 150: e65-e71.

123 Watkin LB, Jessen B, Wiszniewski W, et al. COPA mutations impair ER-Golgi transport and cause hereditary autoimmune-mediated lung disease and arthritis. Nat Genet 2015; 47: 654-660.

124 Mercier S, Kury S, Shaboodien G, et al. Mutations in FAM111B cause hereditary fibrosing poikiloderma with tendon contracture, myopathy, and pulmonary fibrosis. Am J Hum Genet 2013; 93: 1100-1107.

125 Hartmannova H, Piherova L, Tauchmannova K, et al. Acadian variant of Fanconi syndrome is caused by mitochondrial respiratory chain complex I deficiency due to a non-coding mutation in complex I assembly factor NDUFAF6. Hum Mol Genet 2016; 25: 4062-4079.

126 Nir V, Ilivitky A, Hakim F, et al. Pulmonary manifestations of prolidase deficiency. Pediatr Pulmonol 2016; 51: 1229-1233. 